\title{
Cc2d1a, a C2 domain containing protein linked to nonsyndromic mental retardation, controls functional maturation of central synapses
}

\author{
Meng Zhao,*1 Jesica Raingo, ${ }^{2}$ Zhijian "James" Chen, ${ }^{1,4}$ and Ege T. Kavalali",3 \\ Departments of ${ }^{1}$ Molecular Biology, ${ }^{2}$ Neuroscience, and ${ }^{3}$ Physiology and ${ }^{4}$ Howard Hughes Medical Institute, University of \\ Texas Southwestern Medical Center at Dallas, Texas
}

Submitted 2 November 2010; accepted in final form 23 January 2011

\begin{abstract}
Zhao M, Raingo J, Chen ZJ, Kavalali ET. Cc2d1a, a C2 domain containing protein linked to nonsyndromic mental retardation, controls functional maturation of central synapses. J Neurophysiol 105: 1506-1515, 2011. First published January 27, 2011; doi:10.1152/jn.00950.2010.$\mathrm{Cc} 2 \mathrm{~d} 1 \mathrm{a}$ is an evolutionarily conserved protein composed of $\mathrm{NH}_{2}$-terminal Drosophila melanogaster 14 domain (DM14) domains and a $\mathrm{COOH}-$ terminal C2 domain. Human patients with homozygotic mutation in the gene suffer from nonsyndromic mental retardation, implying that Cc2d1a functions in the central nervous system. To examine the physiological role of the Cc2d1a, we generated and analyzed Cc2d1a knockout (KO) mice. Cc2d1a KO mice die soon after birth, apparently because of their inability to breathe. Histological analysis of $\mathrm{Cc} 2 \mathrm{~d} 1 \mathrm{a} \mathrm{KO}$ animals did not identify any structural defects in the peripheral respiratory apparatus. However, functional analysis of synapses formed between Cc2d1adeficient cortical neurons revealed a robust increase in the pace of maturation of evoked synaptic responses as well as synaptic vesicle trafficking. This synaptic anomaly was rescued by reintroducing fulllength $\mathrm{Cc} 2 \mathrm{~d} 1 \mathrm{a}$ but not $\mathrm{C} 2$-domain-deletion mutant, underscoring the functional importance of $\mathrm{C} 2$ domain. Our data suggest that Cc2d1a is required for mouse survival and performs essential function in controlling functional maturation of synapses.
\end{abstract}

lethal (2) giant discs; synapse development; synaptic transmission

CC2D1A GENE FAMILY CONSISTS of two homologs, CC2D1A and $\mathrm{CC} 2 \mathrm{D} 1 \mathrm{~B}$, in human and mouse. Recent studies have suggested several potential activities for Cc2d1a. These include functions as a scaffold in the 3-phosphoinositide-dependent protein kinase/Akt pathway (Aki-1) (Nakamura et al. 2008) and in binding to DNA and repressing transcription (Freud-1) (Ou et al. 2003; Rogaeva et al. 2007). Cc2d1a strongly activates the transcription factor NF- $\kappa \mathrm{B}$ through several components of the canonical NF- $\kappa$ B activating pathway (Zhao et al. 2010). However, the physiological function of Cc2d1a remains unknown. Cc2d1a was independently discovered in Drosophila, where it is called lethal (2) giant discs, L(2)gd. Its mutations induce hyperplastic overgrowth and duplication of Drosophila imaginal discs (Buratovich and Bryant 1995). The effects of L(2)gd mutants are mediated by the strong activation of Notch. L(2)gd restricts Notch activity during endocytosis of the Notch receptor. Because Notch pathway is important for organ development, L(2)gd mutants have defects in multiple organs, including wings, eyes, and bristles (Klein 2003; Childress et al. 2006; Gallagher and Knoblich 2006; Jaekel and Klein 2006).

In humans, Cc2d1a has also been linked to autosomal recessive nonsyndromic mental retardation. A mutation in the

\footnotetext{
* M. Zhao and J. Raingo contributed equally to the paper.

Address for reprint requests and other correspondence: E. Kavalali, UT Southwestern Medical Ctr., Dallas, TX 75390 (e-mail: Ege.Kavalali@UTSouthwestern. edu; e-mail for Z. J. Chen: Zhijian.Chen@UTSouthwestern.edu).
}

gene has been identified in nine consanguineous families with severe mental retardation. This mutation results in a large genomic deletion of 3,589 nucleotides, creating a truncated protein; therefore, it may present a loss-of-function effect (Basel-Vanagaite et al. 2006).

In the present study, we generated and analyzed knockout (KO) mice for Cc2d1a to study its function. Cc2d1a-deficient mice die right after birth, apparently because of their inability to breathe. Although histological analysis identified no major morphological changes, Cc2d1a-deficient neurons showed developmentally specific alterations in evoked neurotransmitter release events during the maturation of synapses. In addition, our data also suggest that $\mathrm{Cc} 2 \mathrm{~d} 1 \mathrm{a}$ regulates the endocytic rate of synaptic vesicles. Taken together, these findings implicate $\mathrm{Cc} 2 \mathrm{~d} 1 \mathrm{a}$ as a developmental regulator of synapse function.

\section{METHODS}

Generation of Cc2dla KO mice. The Cc2d1a targeting construct was built using the pN-Z-TK2 vector (kindly provided by $\mathrm{K}$. Song), which contains a nuclear LacZ (nLacZ) cassette. The neomycin-resistance gene flanked by loxP sites were cloned from PGKneolox2DTA.2 (kindly provided by M. Tallquist) into the targeting vector downstream of nLacZ cassette. The 4.2-kb 5' arm and 2.2-kb 3' arm were amplified using PCR and confirmed by sequencing. The $\mathrm{nLacZ}$ and neomycin cassette were fused in frame to exon 1 following the first two amino acids of Cc2d1a, placing the $\mathrm{LacZ}$ reporter gene under the control of the endogenous $\mathrm{Cc} 2 \mathrm{~d} 1 \mathrm{a}$ promoter. The targeting construct was linearized and electroporated into $129 \mathrm{SvEv}$-derived embryonic stem (ES) cells. With the use of Southern blot analysis with $5^{\prime}$ and $3^{\prime}$ probes, three Cc2d1a-targeted ES clones were identified and used for blastocyst injection. The resulting chimeric mice were bred to $\mathrm{C} 57 \mathrm{BL} / 6$ to obtain germline transmission of the mutant allele. All results reported here were performed on littermate mice derived from heterozygous breedings. All experimental protocols and techniques were approved by the University of Texas Southwestern Medical Center Institutional Animal Care and Use Committee.

Southern blot analysis and PCR genotyping. DNA sequences for PCR genotyping primers are as follows: 5'-GTGCGAGGCCAGAGGCCACTTGTG -3'; 5' -GACCCTGAGAGAGCTCCTGAGAGC '; and 5' TTTCCCACCTCTTCTGGCCCAGAGG -3'. Southern blot probes were generated by PCR using the following primer sets: 5' probe forward, 5'-CCA AGC ATTCAAATAGATGAGCCAACGGCAG -3', reverse, 5'-ATTCCCCCTCCCAGGCAAACAGGAGCCAAG -3'; and 3' probe forward, 5'-AGGATCATGCCTGACTTTGAGGTGG -3', reverse, 5' TCTTGTCCTGGTCCACATGATCCAT-3'. In brief, tail genomic DNA was digested with BamHI and analyzed using a standard Southern blot protocol. The $20-\mu \mathrm{l}$ PCR genotyping reaction contained $1 \mu \mathrm{l}$ of tail DNA as template, $0.5 \mu \mathrm{M}$ of each primer, and $10 \mu \mathrm{l} 2 \times$ GoTaqGreen mix (Promega). An annealing temperature of $55^{\circ} \mathrm{C}$ was used, and the PCR products were analyzed by electrophoresis on a $2 \%$ agarose gel. 
Immunoblotting analysis. Tissues were homogenized in extraction buffer (20 mM Tris. $\mathrm{HCl}$ pH 7.50, $100 \mathrm{mM} \mathrm{NaCl,} \mathrm{10 \%} \mathrm{Glycerol,} \mathrm{0.5 \%}$ Triton-X 100, and protease inhibitor cocktail from Roche) and subjected to centrifugation at $12,000 \mathrm{~g}$ for $10 \mathrm{~min}$ at $4^{\circ} \mathrm{C}$. The soluble fractions were collected, and $40 \mu \mathrm{g}$ of proteins were loaded onto $7 \%$ SDS-PAGE gels; immunoblotting was then performed according to standard protocols. The antibody against human CC2D1A was described in Zhao et al. 2010.

Histology and in situ hybridization. E18.5 embryos were harvested from timed mating and fixed in $4 \%$ paraformaldehyde. The skin was removed to facilitate fixation. Staining with hematoxylin and eosin was performed by Molecular Pathology Core Laboratory at University of Texas Southwestern using standard procedures. Whole brain was dissected from E18.5 embryos and fixed overnight in diethyl pyrocarbonatetreated $4 \%$ paraformaldehyde. Riboprobes were labeled with ${ }^{35} \mathrm{~S}-\mathrm{UTP}$ by using the MAXIscript in vitro transcription kit (Ambion). The template was amplified by PCR using primers $5^{\prime}$-tagagaaactgaaaggccaag- $3^{\prime}$ and $5^{\prime}$-agccaactgggaagtagcttg- $3^{\prime}$ and cloned into pcDNA3 under the T7 promoter. In situ hybridization of sectioned tissues was performed by Molecular Pathology Core Laboratory at University of Texas Southwestern as previously described (Vega et al. 2004).

Subcellular fractionation of synaptosomes using a Percoll gradient. Synaptosomes from adult mouse forebrain were purified as described previously (Khvotchev and Sudhof 2004). All procedures were performed at $4^{\circ} \mathrm{C}$. The tissue were dissected and homogenized in a glass douncer (Wheaton Science Products) in buffer A $(0.32 \mathrm{M}$ sucrose, 5 mM HEPES-NaOH, pH 7.4, 0.1 mM EDTA). Homogenate was fractionated by low-speed centrifugation (1,000 $\mathrm{g}$ for $10 \mathrm{~min})$, and the low-speed supernatant (S1) was centrifuged at $14,500 \mathrm{~g}$ for 20 min to obtain the crude synaptosome fraction (P2). The P2 pellet was resuspended in $8.5 \%$ Percoll in buffer containing $0.25 \mathrm{M}$ sucrose, 5 mM HEPES-NaOH, pH 7.4, and 0.1 mM EDTA and layered on top of a $12 \% / 20 \%$ Percoll step gradient in the same buffer. After centrifugation at $18,000 \mathrm{~g}$ for $30 \mathrm{~min}$, synaptosomes were recovered from the $12 \% / 20 \%$ Percoll interface. Percoll was removed by adding 30 volumes of buffer A and centrifugation at 18,000 $\mathrm{g}$ for $15 \mathrm{~min}$. Purified synaptosomes (P4) were resuspended in buffer A.

Whole-mount staining of diaphragm muscle. Muscle samples were fixed in $2 \%$ paraformaldehyde in $0.1 \mathrm{M}$ phosphate buffer $(\mathrm{pH} 7.3)$ at $4{ }^{\circ} \mathrm{C}$ overnight, blocked in dilution buffer $(500 \mathrm{mM} \mathrm{NaCl}, 0.01 \mathrm{M}$ phosphate buffer, $3 \%$ BSA and $0.01 \%$ thimerosal), and then incubated with primary antibodies. The primary antibodies for Syntaxin and Synaptotagmin-2 were from Dr. Weichun Lin (University of Texas Southwestern). Samples were then incubated with fluorescein isothiocyanate-conjugated secondary antibody and Texas-Red-conjugated $\alpha$-bungarotoxin (2 nM, Molecular Probes), washed with PBS, and mounted in $90 \%$ glycerol, and $10 \%$ Tris buffer $(\mathrm{pH} 8.5,10 \mathrm{mM})$ containing $n$-propyl gallate $(20 \mathrm{mM})$ to reduce photobleaching (Giloh and Sedat 1982). Fluorescence images of acetylcholine receptors (AChR) clusters were generated from confocal images acquired with identical, subsaturating gains.

Cortical primary neuronal cultures. The cortices were dissected from the brains of embryonic day 18.5 (E18.5) mice, dissociated by trypsin digestion, and plated on circular glass coverslips coated with poly-lysine. The cortical neurons were maintained in MEM medium (Invitrogen) supplemented with B-27 (Invitrogen), L-glutamine, 0.5\% glucose, $5 \%$ fetal bovine serum, and cytosine-arabinoside (SigmaAldrich) (Kavalali et al. 1999). The cultures were used for experiments as indicated.

Lentiviral infection. Lentiviral constructs were cotransfected with plasmids for viral enzymes and envelope proteins into human embryonic kidney 293 cells using a transfection system (FuGENE6; Roche) according to the manufacturer's specifications, and lentivirus containing culture medium was harvested 3 days later and centrifuged at 1,000 revolution/min for $10 \mathrm{~min}$. The supernatant was immediately used for infection or frozen in liquid nitrogen and stored at $-80^{\circ} \mathrm{C}$.
Cortical cultures were infected on day 4 by adding $200 \mu$ l of viral suspension to each well.

Electrophysiology. Synaptic activity was recorded from cortical pyramidal neurons using the whole cell voltage-clamp technique. Data were acquired using an Axopatch 200B amplifier and Clampex 9.2 software (Molecular Devices). Recordings were filtered at $2 \mathrm{kHz}$ and sampled at $200 \mu \mathrm{s}$. A modified Tyrode's solution [containing the following (in $\mathrm{mM}$ ): $150 \mathrm{NaCl}, 4 \mathrm{KCl}, 2 \mathrm{MgCl}_{2}, 2 \mathrm{CaCl}_{2}, 10$ glucose, and 10 HEPES, $\mathrm{pH}$ 7.4] was used as external bath solution. To isolate inhibitory postsynaptic currents (IPSCs), external bath solution contained $10 \mu \mathrm{M}$ 6-cyano-7-nitroquinoxaline-2,3-dione (CNQX) and 50 $\mu \mathrm{M}$ (2R)-amino-5-phosphonovaleric acid (APV) to block glutamatergic currents. Excitatory postsynaptic currents (EPSCs) were isolated with the addition of $50 \mu \mathrm{M}$ picrotoxin to block GABAergic currents. All recordings of spontaneous miniature IPSCs were performed in 1 $\mu \mathrm{M}$ tetrodotoxin to block action potential firing. The pipette internal solution contained the following (in $\mathrm{mM}$ ): $115 \mathrm{Cs}-\mathrm{MeSO}_{3}, 10 \mathrm{CsCl}, 5$ $\mathrm{NaCl}, 10$ HEPES, 0.6 EGTA, 20 tetraethylammonium-Cl, $4 \mathrm{Mg}$-ATP, $0.3 \mathrm{Na}_{3} \mathrm{GTP}, \mathrm{pH}$ 7.35, and $10 \mathrm{QX}-314$ (300 mOsm). To elicit evoked responses, electrical stimulation was delivered through parallel platinum electrodes in modified Tyrode's solution (duration, $1 \mathrm{~ms}$; amplitude, $20 \mathrm{~mA}$ ). All electrophysiology experiments were performed in at least three independent neuronal cultures.

Fluorescence imaging. The synaptophysin-pHluorin construct containing two pHluorins was a generous gift from Drs. Y. Zhu and C. F. Stevens (The Salk Institute, La Jolla, CA). Cortical cultures were infected with lentivirus expressing the synaptophysin-pHluorin construct on day 4, and imaging experiments were performed on day 14 at room temperature. A modified Tyrode's solution containing $2 \mathrm{mM}$ $\mathrm{Ca}^{2+}, 10 \mu \mathrm{M}$ CNQX, and $50 \mu \mathrm{M}$ APV was used in all experiments. Baseline images were obtained every second for $30 \mathrm{~s}$, and cultures were stimulated with field stimulation.

\section{RESULTS}

Deletion of Cc2dla is lethal. We ablated Cc2d1a gene in mice by homologous recombination to investigate the function of $\mathrm{Cc} 2 \mathrm{~d} 1 \mathrm{a}$ in vivo. The targeting strategy resulted in the deletion of amino acids 3-349 encoded by exons 1-10 of the gene and insertion of a LacZ reporter gene in frame with amino acid 2 and a neomycin selection cassette flanked by loxP sites $3^{\prime}$ to LacZ coding sequences (Fig. 1A). We maintained three independent lines (1AKO-A, 1AKO-B, 1AKO-C) originating from three different ES cell electroporations. The phenotypes of the three lines are identical, confirming that it is the result of the Cc2d1a deletion and not that of another mutation introduced by ES cell manipulation. For this study, we used mice from the $1 \mathrm{AKO}-\mathrm{A}$ line. The deletion of $\mathrm{Cc} 2 \mathrm{~d} 1 \mathrm{a}$ was verified by PCR and Southern and Western blotting (Fig. 1, $B$ and $C$ ).

Heterozygous mice are viable and fertile, whereas Cc2d1adeficient mice die within a few minutes after birth. Analysis of embryos, however, showed a normal Mendelian ratio of genotypes up to embryonic day (E)18.5, right before birth, indicating that lethality occurred at birth (Fig. 1D). KO mice showed no spontaneous movement or breathing activity but displayed weak and uncoordinated movement in response to mild pinches on their tails or legs, suggesting that the neural circuits for reflective responses are intact in these mutants. Therefore, a severe postnatal respiratory failure may be the cause of lethality.

Expression of Cc2dla is enriched in the brain. A previous study demonstrated that $\mathrm{Cc} 2 \mathrm{~d} 1 \mathrm{a}$ is highly expressed in developing mouse brain (Basel-Vanagaite et al. 2006). However, the tissue expression pattern of $\mathrm{Cc} 2 \mathrm{~d} 1 \mathrm{a}$ has not been documented. 
Fig. 1. Targeting the Cc2d1a locus. A: strategy to generate the Cc2d1a-deficient allele. The domain structure of the mouse (m) Cc2d1a protein, first 3 ATG sites, corresponding exonal structure, targeting vector, and targeted allele are shown. LacZ cassette and loxP-Neo-loxP replace exons 1-10. Positions of the probe used for Southern analysis are shown. $B$ : genomic DNA from mice of the indicated genotypes was analyzed by Southern blot. $C$ : protein extracted from E14.5 myocyte enhancer factor cells was analyzed by immunoblotting using CC2D1A-specific antibody. Genomic DNA from embryonic day 14.5 (E14.5) embryos was analyzed by genotyping PCR.

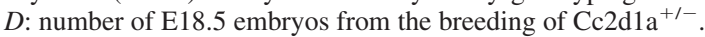
WT, wild-type; KO, knockout; IB: immunoblot; Het, heterozygous mice.

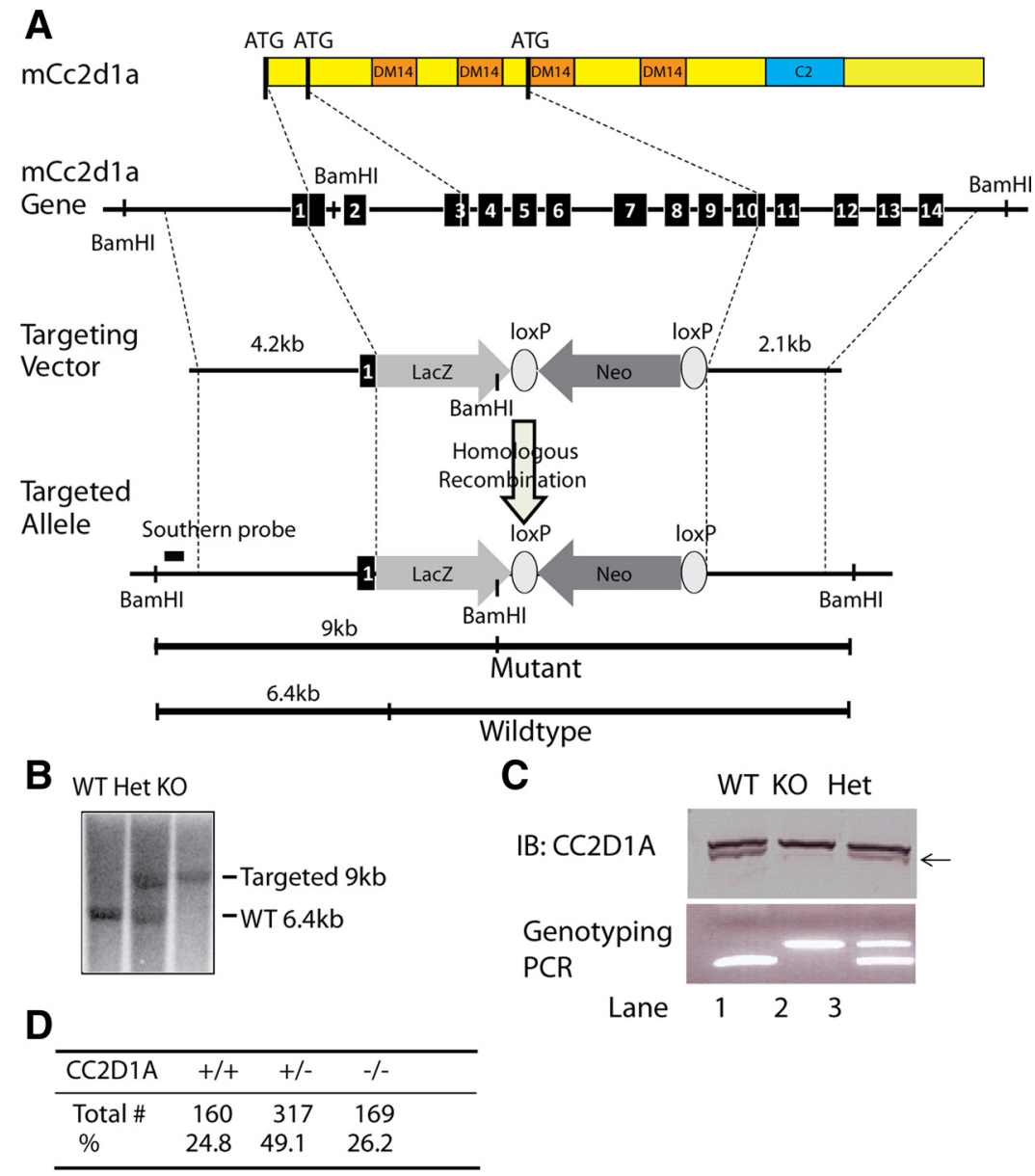

Therefore, we carried out immunohistochemical staining using Cc2d1a antibody. Although the antibody is able to recognize Cc2d1a on Western blotting, it did not specifically label endogenous protein in its native form. We isolated different tissue from E18.5 wild-type embryos, extracted protein from tissues using lysis buffer containing $0.5 \%$ Triton-X 100, and compared expression profile of Cc2d1a. As illustrated in Fig. 2A, Cc2d1a is enriched in the brain. To determine the spatial expression pattern of Cc2d1a in the brain, we carried out in situ hybridization in E18.5 embryos (Fig. 2B). The ${ }^{35} \mathrm{~S}$-labeled probe corresponds to the $5^{\prime}$ coding sequence that is replaced in $\mathrm{KO}$. No signal from $\mathrm{KO}$ indicates that the probe specifically detects endogenous $\mathrm{Cc} 2 \mathrm{~d} 1 \mathrm{a}$ mRNAs. In agreement with a previous report $(\mathrm{Ba}-$ sel-Vanagaite et al. 2006), Cc2d1a mRNA was found throughout the mouse brain. From coronal sections at the bottom of Fig. $2 B$, it seems that the signal from the olfactory bulb is the strongest and that the mRNA expression is enriched in the gray matter, including cortical plate of neocortex, hippocampus, basal ganglia, and hypothalamus.

In an attempt to identify the subcellular localization of $\mathrm{Cc} 2 \mathrm{~d} 1 \mathrm{a}$ in the brain, we performed biochemical fractionation of forebrains from adult mice using a Percoll step gradient (Khvotchev and Sudhof 2004). As shown in Fig. $2 C$, synaptic proteins including $N$-methyl-D-aspartate receptor-1, Synaptotagmin-1, Synaptophysin-1, and Syntaxin-1 are enriched in synaptosome fractions, whereas a cytosolic protein Traf6 is in the cytosol fraction. Cc2d1a is enriched in synaptosome fractions, suggesting that $\mathrm{Cc} 2 \mathrm{~d} 1 \mathrm{a}$ may localize to the synapse.

Anatomy of Cc2dla KO mice is normal. Loss of the Drosophila Cc2d1a orthologs L(2)gd causes massive overproliferation of imaginal disc cells, extended larval life, and lethality during pupae stage (Agrawal et al. 1995; Buratovich and Bryant 1997; Klein 2003). These earlier findings raise the possibility that Cc2d1a-deficient mice suffer from major developmental abnormalities that cause lethality. To identify such abnormalities, we examined the morphology of E18.5 Cc2d1a-deficient embryos by hematoxylin and eosin staining. These experiments did not reveal a major anatomical defect in Cc2d1a-deficient mice. In particular, the heart, lung, and brain were apparently normal (Fig. 3A).

Because $\mathrm{KO}$ mice do not breathe after birth, we asked whether the formation of neuromuscular junctions at the diaphragm was affected in the absence of Cc2d1a. We carried out immunofluorescence staining using Syntaxin-specific antibody to label phrenic nerves at the diaphragm. As shown in Fig. 3B, the branching pattern was not significantly altered in Cc2d1a KO mice. We also stained diaphragm muscles with Texas-Red conjugated $\alpha$-bungarotoxin to label postsynaptic nicotinic AChRs and with Synaptotagmin-2-specific antibody to label nerve terminals (Fig. 3C). The distribution of AChRs in the wild-type and Cc2d1a-deficient muscles were similar, and the nerve terminals formed juxtaposition with AChR clusters (Fig. $3 C$ ). Therefore, neuromuscular junctions were established in the absence of $\mathrm{Cc} 2 \mathrm{~d} 1 \mathrm{a}$. 
A
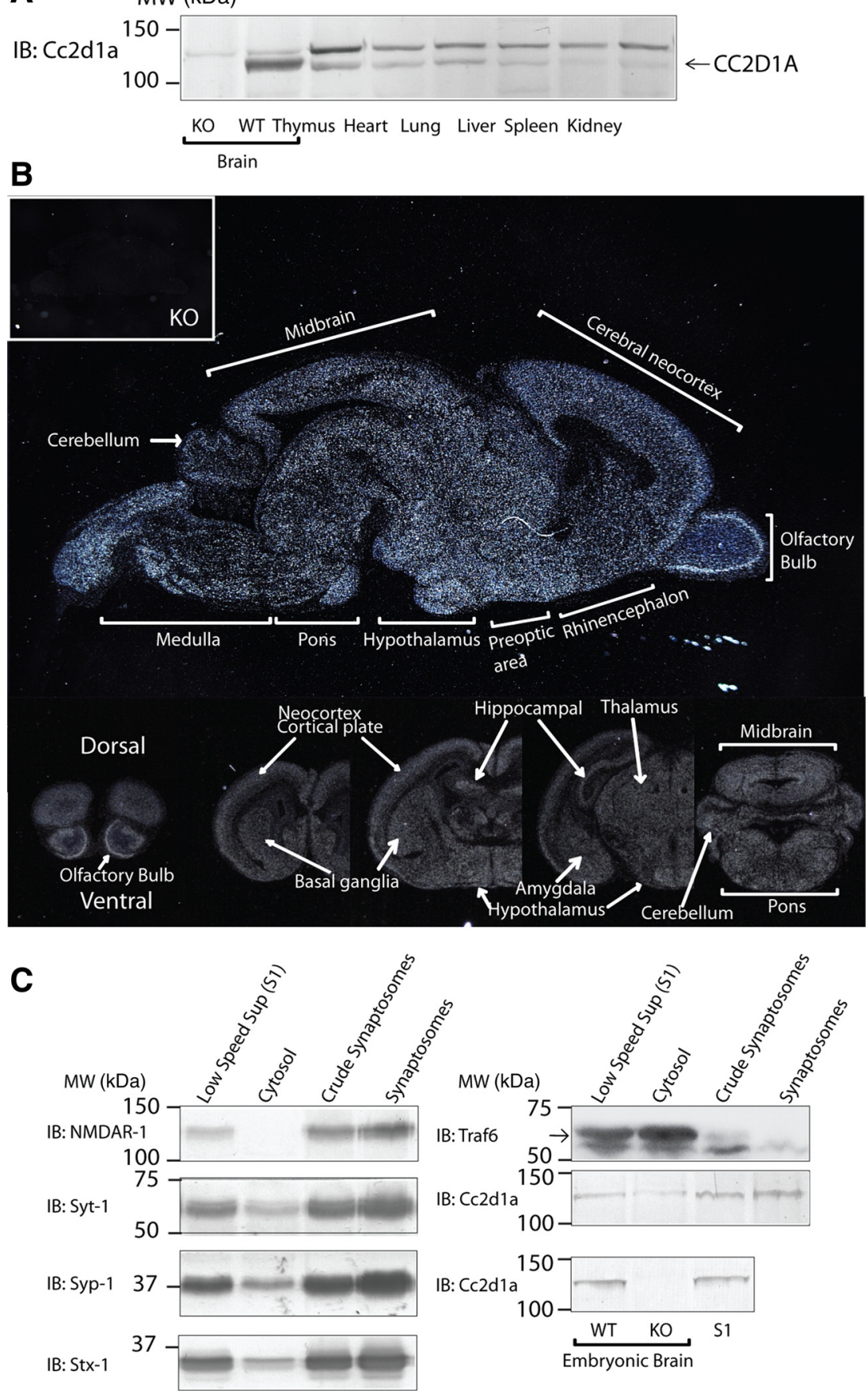

Fig. 2. Expression of $\mathrm{Cc} 2 \mathrm{~d} 1 \mathrm{a}$ is enriched in the brain. $A$ : protein extracted from different tissues of E18.5 embryos were examined by Western blot using CC2D1A antibody. $B$ : detection of $\mathrm{Cc} 2 \mathrm{~d} 1 \mathrm{a}$ transcripts by in situ hybridization. Inset: sagittal section of a $\mathrm{KO}$ embryo. In the center is a sagittal section of a WT embryo. At the bottom are coronal sections of a WT embryo. $C$ : subcellular fractionation of adult forebrains using Percoll step gradient. As described in METHODS, brain homogenate was centrifuged at $1,000 \mathrm{~g}$ to obtain low-speed supernatant (Sup) (S1) or 100,000 $g$ to obtain cytosol. Low-speed supernatant was centrifuged at $14,500 \mathrm{~g}$, and the pellet provided crude synaptosomes. Synaptosome fraction banded at the interface of $12 \% / 20 \%$ Percoll. The same amount of protein from each fraction was used for Western blotting. Protein extract from embryonic brain tissues of WT and KO mice were used to verify the specificity of the Cc2d1a antibody. MW, molecular weight; NMDAR-1, $N$-methyl-D-aspartate receptor-1; Syt-1, Synaptotagmin-1; Syp-1, Synaptophysin-1; Stx-1, Syntaxin-1.
Developmental increase in the amplitudes of evoked IPSCs in Cc2dla-deficient synapses. Histological analysis of Cc2d1a $\mathrm{KO}$ animals did not show any defects in the peripheral respiratory apparatus, including the lungs, intercostal musculature, or in the nerve innervation pattern of the diaphragm. These observations point to a possible defect that may originate from an alteration in central synaptic function. Neurons that control the breathing are found in many regions of the neuraxis from cerebral cortex through the brainstem and spinal cord (Harper et al. 1998). Given that Cc2d1a expression is enriched in the brain, especially in the cortex, we decided to focus on this part of the brain. In addition, because L(2)gd, Drosophila ortholog of $\mathrm{Cc} 2 \mathrm{~d} 1 \mathrm{a}$, regulates endocytosis, we hypothesized that Cc2d1a may be involved in neurotransmitter release by regulating synaptic exocytosis and endocytosis.

We wanted to test the basic synaptic properties of cortical neurons; however, because $\mathrm{Cc} 2 \mathrm{~d} 1 \mathrm{a} \mathrm{KO}$ mice die at birth, we used cultured neurons from the neocortex of E18.5 embryos in vitro. Under these controlled conditions, neurons have sufficient time to develop and form extensive synaptic connections (Atasoy et al. 2007), allowing us to study the role of Cc2d1a in neurotransmission.

To examine the properties of basic synaptic transmission in Cc2d1a KO synapses, we performed whole cell voltage-clamp recordings in cultured cortical pyramidal neurons. We analyzed action potential-evoked IPSCs induced by field stimula- 
A

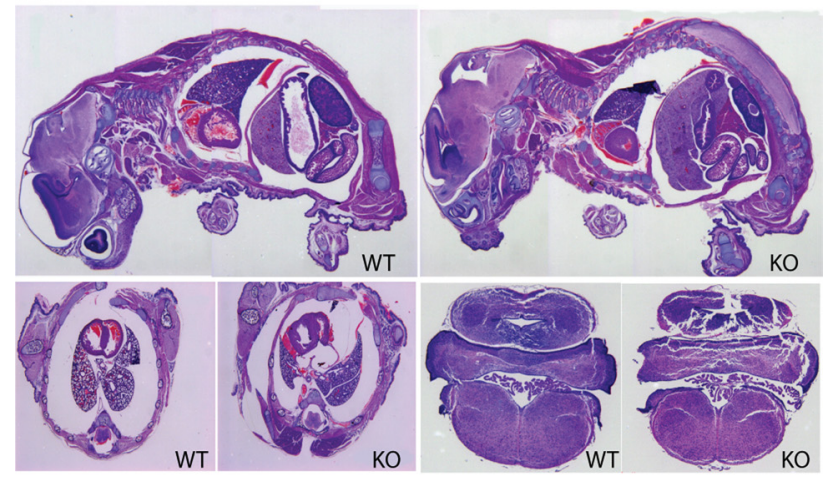

B

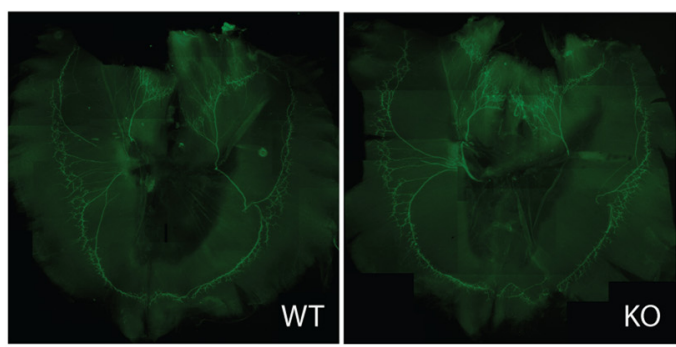

C

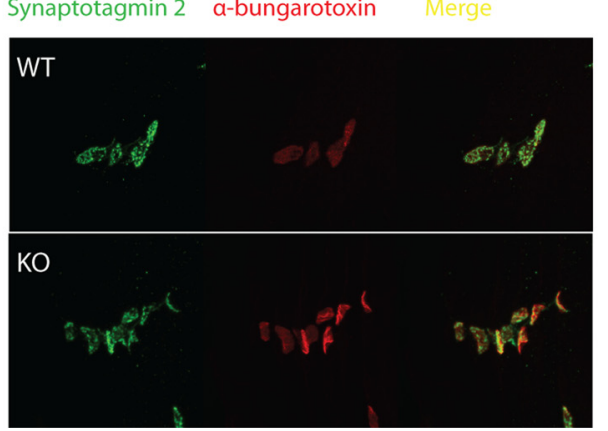

Fig. 3. Anatomy of $\mathrm{Cc} 2 \mathrm{~d} 1 \mathrm{a} \mathrm{KO}$ mice is normal. A: top: sagittal sections of WT and $\mathrm{Cc} 2 \mathrm{~d} 1 \mathrm{a} \mathrm{KO}$ embryos at E18.5. Bottom, left: transverse sections through the heart and lung. Bottom, right: coronal sections through the brain. B: wholemount embryonic diaphragm muscles (E18.5) were immunostained with antiSyntaxin antibody. $C$ : confocal images of single endplates from whole-mount diaphragm muscle at E18.5, double-labeled with Syt-2 antibody (green) and Texas-red conjugated $\alpha$-bungarotoxin (red).

tion. Cultured cortical neurons were examined at three different developmental stages, 7, 14 and 21 days in vitro (DIV). As shown in Fig. 4A, we found that at 7 DIV, when most synapses from control (wild-type or heterozygous) neurons showed limited evoked neurotransmitter release (Mozhayeva et al. 2002), synapses from Cc2d1a-deficient neurons were more responsive to action potential stimulation. At 14 DIV, IPSCs from both control and $\mathrm{KO}$ groups were higher although the difference between the two groups of synapses remained statistically significant (Fig. $4 B$ ). Interestingly at 21 DIV, the responsiveness of IPSCs to action potential became indistinguishable from controls (Fig. 4C). In all three stages, when we normalized IPSC amplitudes with respect to the maximum IPSC, we did not detect a significant difference between $\mathrm{KO}$ and control synapses, indicating that the increase in the size of the evoked IPSC was not attributable to an alteration in the properties of short-term dynamics of neurotransmitter release (Fig. 4, $A-C$, right). We also examined EPSCs induced by field stimulation at 14 DIV and found significantly larger ( $\sim 2$-fold) evoked EPSCs originating from Cc2d1a-deficient synapses (Fig. 5A). This observation parallels our findings on developmental augmentation of evoked IPSCs. In both control and Cc2d1a KO neurons, we detected swift synaptic depression and recovery in response to repetitive stimulation. Hypertonic sucrose-evoked responses, on the other hand, showed only a small increase that did not reach significance (Fig. 5, $B$ and $C$ ). These results show that loss-of-function phenotype of $\mathrm{Cc} 2 \mathrm{~d} 1 \mathrm{a}$ affects both inhibitory and excitatory synapses. Taken together, these findings suggest a developmental impact of $\mathrm{Cc} 2 \mathrm{~d} 1 \mathrm{a}$ in the functional synapse maturation process.

Properties of spontaneous miniature IPSCs in Cc2dladeficient synapses. To gain further insight to the synaptic mechanisms that may underlie these alterations in evoked neurotransmission, in the next set of experiments, we focused on the properties of spontaneous miniature IPSCs as well as neurotransmitter release driven by hypertonic sucrose stimulation. Analysis of the properties of spontaneous miniature IPSCs (mIPSCs) recorded under resting conditions at 7 DIV did not reveal a significant difference in their frequencies or amplitudes between the Cc2d1a-deficient neurons and controls (Fig. 6A). In contrast, at 14 DIV the frequency of mIPSCs recorded from $\mathrm{Cc} 2 \mathrm{~d} 1 \mathrm{a}-\mathrm{deficient}$ neurons lagged significantly behind their control counterparts although the amplitudes of mIPSCs remained comparable under the two conditions (Fig. $6 B$ ). Finally, at 21 DIV the frequencies of mIPSCs were again comparable between $\mathrm{KO}$ and control cultures, whereas mIPSC amplitudes showed a small but significant increase in Cc2d1adeficient neurons. Taken together, these results suggest that the developmental increase seen in evoked IPSC amplitudes after loss of Cc2d1a (Fig. 4) is not attributable to a substantial increase in the number of postsynaptic receptors, as the amplitudes of individual mIPSC do not show a dramatic change. In addition, these results also indicate that loss of $\mathrm{Cc} 2 \mathrm{~d} 1 \mathrm{a}$ does not result in a major increase in the numbers of synapses that could give rise to the augmentation of evoked IPSCs. If anything, at 14 DIV the frequency of mIPSCs shows a reduction in contrast to the increase in evoked IPSC amplitudes recorded at the same developmental stage.

To test the question whether the number of functional synapses or the size of the readily releasable pool of vesicles are altered after loss of $\mathrm{Cc} 2 \mathrm{~d} 1 \mathrm{a}$, we next recorded neurotransmitter release evoked in response to perfusion of a hypertonic solution (with addition of $500 \mathrm{mOsm}$ sucrose to normal extracellular solution). As in excitatory neurotransmission results shown in Fig. 5, these experiments did not uncover a significant difference between control and Cc2d1a-deficient neurons, indicating that the increase in the size of evoked IPSCs (as well as EPSCs) were presumably attributable to a specific alteration in action potential-evoked release (Fig. 6B).

Synaptic vesicle recycling in Cc2dla KO synapses. In the next set of experiments, we aimed to examine whether loss of Cc2d1a resulted in a specific defect in presynaptic function. For this purpose, we monitored the fusion and retrieval of synaptic vesicles in response to stimuli using synaptophysinpHluorin, where two $\mathrm{pH}$-sensitive green fluorescent proteins are fused to synaptophysin (Zhu et al. 2009). SynaptophysinpHluorin is quenched by the acidic $\mathrm{pH}$ inside a vesicle, but fluorescence emission increases when the interior of the vesicle connects with the external medium, followed by a rapid decline when the vesicle is retrieved and its interior reacidified. When 

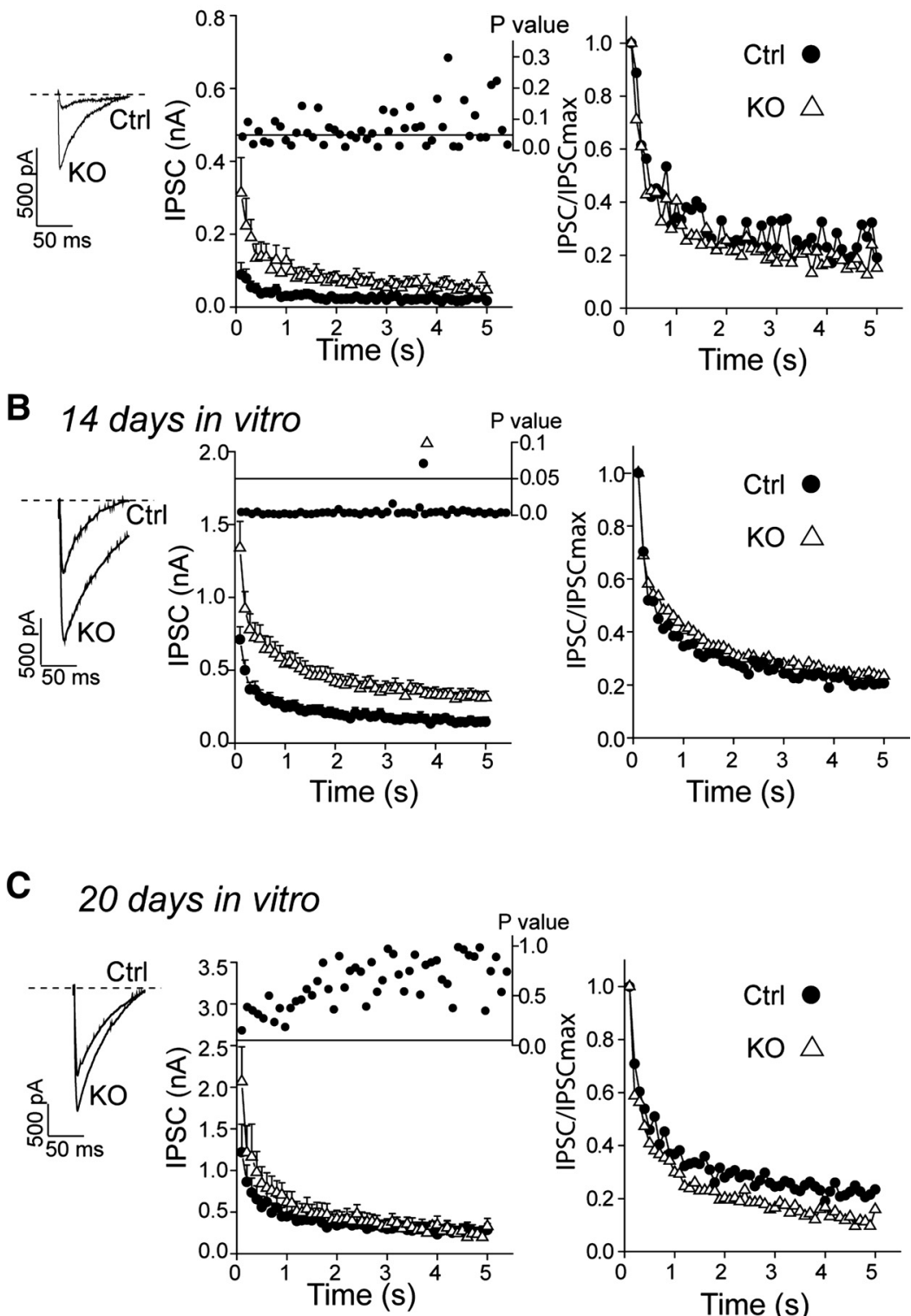

$P$ value

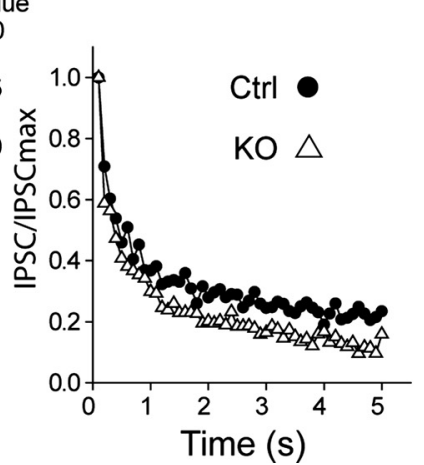

Fig. 4. Evoked GABAergic responses in $\mathrm{Cc} 2 \mathrm{~d} 1 \mathrm{a}$ KO neurons. $A$ : representative traces (inset and left) and average synaptic responses (right) from 7 days in vitro (DIV) cortical neurons. Evoked inhibitory postsynaptic currents (IPSCs) were recorded in response to maximal field stimulation applied for $5 \mathrm{~s}$ at 10 $\mathrm{Hz}$. The mean amplitudes of GABAergic responses (IPSCs) under the inhibition of glutamatergic responses were measured at each stimulation (Control, $n=14$; KO, $n=13$ ). Representative traces on the left depict the first responses from control or Cc2d1a KO neurons. $P$ values indicating the statistical significance of the difference between control and Cc2d1a KO traces are plotted above the average values. When IPSCs were normalized with respect to the first response (IPSCmax) the resulting traces did not reveal a kinetic difference between the control and $\mathrm{KO}$ conditions. $B$ : cortical neurons at 14 DIV were examined as in $A$ (Control, $n=20$; KO $n=22$ ). The difference between the average traces is significant $(P<0.05)$ throughout the stimulation period. Inset: representative traces (scale bar 1 s, $0.2 \mathrm{nA}$ ). $C$ : cortical neurons at 21 DIV were examined as in $A$ (Control, $n=6$; KO, $n=7$ ). Inset: representative traces (scale bar $1 \mathrm{~s}, 1 \mathrm{nA}$ ). Note that control and $\mathrm{KO}$ groups do not show a clear difference at this stage. In all cases, the normalized IPSC values (shown on the right) reveal no differences in the decay kinetics between the groups at 7,14 or 21 DIV. we applied electrical stimulation at $20 \mathrm{~Hz}$ for a duration of 20 $\mathrm{s}$, we did not detect a major change in the overall kinetics of fluorescent responses in Cc2d1a-deficient neurons, as shown by the ascending phase in Fig. $7 A$. This observation suggests that there is no significant impact of $\mathrm{Cc} 2 \mathrm{~d} 1 \mathrm{a}$ on vesicle retrieval during stimulation, which is consistent with the lack of alterations in the kinetics of short-term synaptic depression in $\mathrm{Cc} 2 \mathrm{~d} 1 \mathrm{a} \mathrm{KO}$. Furthermore, this observation also indicates that loss of $\mathrm{Cc} 2 \mathrm{~d} 1 \mathrm{a}$ does not impact release kinetics per synapse but augments the number of synapses that respond to brief stimulation, which is a key hallmark of transition of the synapses to functional maturity (Mozhayeva et al. 2002; Shen et al. 2006; Akhtar et al. 2009). In contrast, the descending phase of the synaptophysin-pHluorin signal showed a markedly faster decline, suggesting an increase in the pace of synaptic vesicle endocytosis in Cc2d1a-deficient neurons, which may contribute to more effective maintenance of neurotransmission in the long term (Sara et al. 2002). In addition, the absolute amplitude of the exocytic response during stimulation showed a small decrease, which did not reach statistical difference (Fig. $7 B, \Delta \mathrm{F}$ during stimulation). This decrease was also reflected in the fluorescent responses seen after the addition of $\mathrm{NH}_{4} \mathrm{Cl}$, which dequenched all acidic vesicles that had not been released (i.e., the total pool of synaptophysin-pHluorin-containing vesicles). However, the fraction of the vesicle pool mobilized during 20-Hz stimulation (compared to the total pool) did not show a major alteration between control and Cc2d1a-deficient synapses. The increase in the rate of fluorescence decay after cessation of stimulation suggests that synaptic vesicles from Cc2d1a KO neurons turn over faster than controls. These findings suggest a specific role for $\mathrm{Cc} 2 \mathrm{~d} 1 \mathrm{a}$ in setting the pace of presynaptic vesicle endocytosis.

The Cc2dla KO phenotype can be partially rescued by Cc2dla. To investigate whether the phenotype of Cc2d1adeficient synapses reflects a direct role of $\mathrm{Cc} 2 \mathrm{~d} 1 \mathrm{a}$ in functional synaptic development vs. a possible indirect effect of loss of $\mathrm{Cc} 2 \mathrm{~d} 1 \mathrm{a}$ on synapse maturation and function, we tested the rescue of the Cc2d1a loss-of-function phenotype by the ex- 
Fig. 5. Evoked excitatory neurotransmission in Cc2d1a KO neurons. A: top: average of excitatory postsynaptic currents (EPSCs) evoked at 14 DIV cultures of control or Cc2d1a stimulation peaks were significantly different between the 2 groups. Inset: representative traces for the initial peaks of control and Cc2d1a KO groups. At the end of the $20-\mathrm{Hz}$ stimulation paradigm, switching to $1-\mathrm{Hz}$ stimulation enabled rapid recovery of both control and Cc2d1a KO synaptic response to their initial values $(n=11$ for control and $n=10$ for KO cells). $B$ and $C$ : representative traces $(B)$ and mean charge movement values integrated during the first 10 $\mathrm{s}$ of 20-s hypertonic stimulation (with the addition of $0.5 \mathrm{M}$ sucrose) of excitatory synapses $(C)$ in control and Cc2d1a $\mathrm{KO}$ neurons. These experiments did not reveal a significant difference between the 2 groups ( $n=6$ for both, $P>0.05$ ). $\mathrm{KO}$ neurons. AP, action potential. Bottom: all the $20-\mathrm{Hz}$
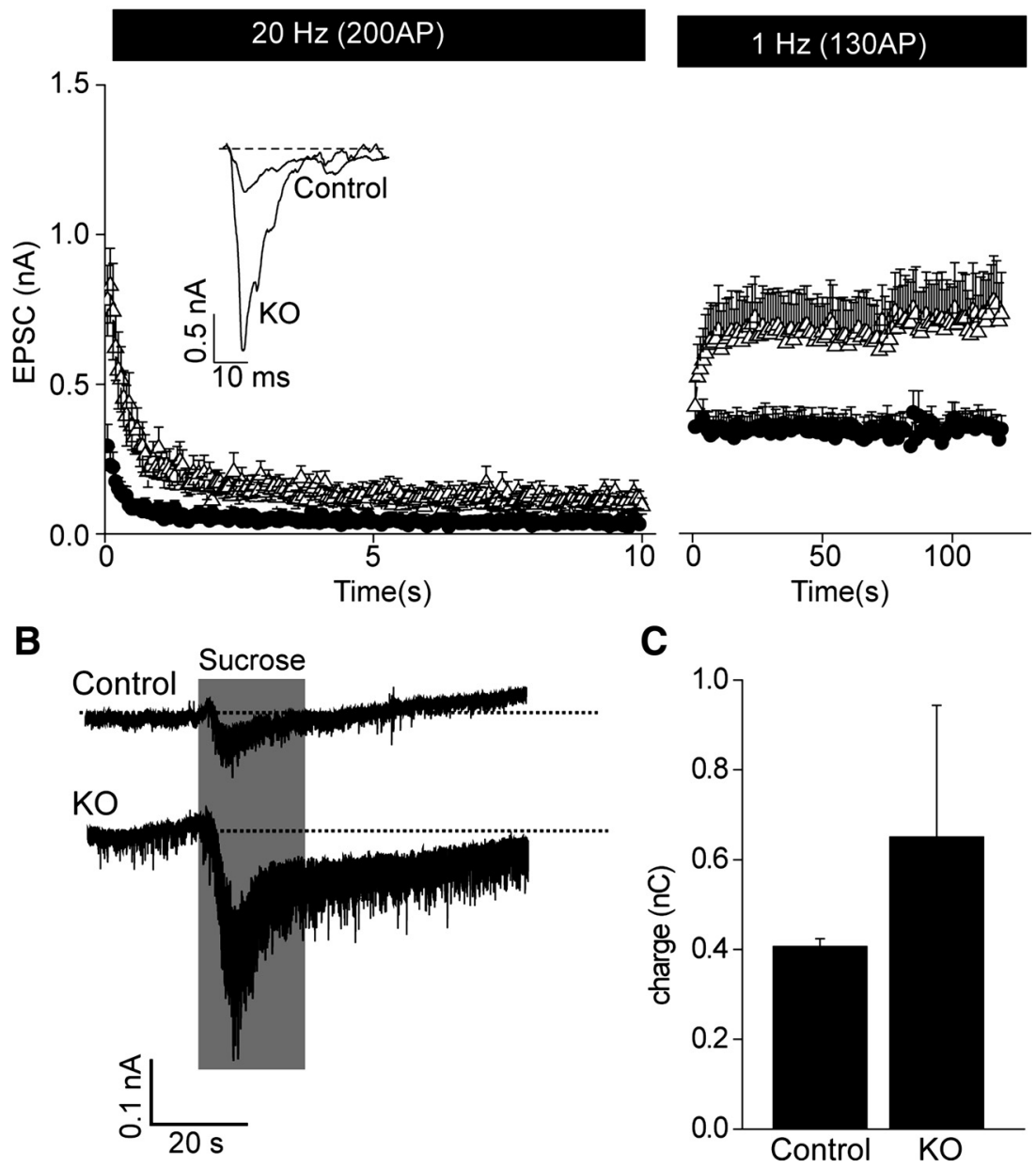

pression of wild-type Cc2d1a in Cc2d1a-deficient neurons. We generated lentiviruses expressing full-length Cc2d1a. Protein expression was confirmed by Western blot (Fig. 8A). Cc2d1adeficient neurons were infected at 4 DIV with these lentiviruses and analyzed at 14 DIV.

Measurements of inhibitory synaptic responses induced by the same field stimulation of 50 action potentials administered at $10 \mathrm{~Hz}$ demonstrated that the Cc2d1a protein partially yet significantly rescued the Cc2d1a-deficiency phenotype (Fig. $8 B$ ). In contrast, $\mathrm{COOH}$-terminus-deletion mutant of $\mathrm{Cc} 2 \mathrm{~d} 1 \mathrm{a}$ was not able to rescue the phenotype, indicating that $\mathrm{C} 2$ domain and the $\mathrm{COOH}$ terminus of the protein are required for synapse regulation exerted by Cc2d1a (Fig. $8 B$ ). Notably the rescue proteins were introduced into the neurons after embryonic development and neuronal specification and while synapse formation and maturation was still in process in vitro. Therefore, the effect of Cc2d1a on neuronal development and synaptic maturation before the expression of the rescue proteins cannot be recovered in this experiment. In addition, although rescue of the synaptic phenotype would suggest that Cc2d1a may act acutely during neurotransmitter release, the time delay between viral infections and synaptic measurements precludes a dissociation of the Cc2dla effects on synapse maturation vs. acute synaptic function. Despite the ineffectiveness of the COOH-terminus-deletion mutant of Cc2d1a (lacking the $\mathrm{C} 2$ domains) in rescuing the synaptic phenotype, the actual role of this domain in neuronal function of $\mathrm{Cc} 2 \mathrm{~d} 1 \mathrm{a}$ remains unclear. In nonneuronal cells, $\mathrm{C} 2$ domain of $\mathrm{Cc} 2 \mathrm{~d} 1 \mathrm{a}$ is required for its effect on NF- $\kappa \mathrm{B}$-dependent transcription (Zhao et al. 2010). C2 domains typically bind to calcium and phospholipids and are present in many synaptic proteins such as synaptotagmins. Five conserved aspartic acids in the $\mathrm{C} 2$ domain are required to bind calcium (Rizo and Sudhof 1998). The alignment of the $\mathrm{C} 2$ domain of $\mathrm{Cc} 2 \mathrm{~d} 1$ a with other canonical $\mathrm{C} 2$ domains showed that $\mathrm{Cc} 2 \mathrm{~d} 1 \mathrm{a}$ does not have all the conserved aspartic acids (data not shown). Furthermore, using the purified $\mathrm{C} 2$ domain of Cc2d1a protein, we did not detect $\mathrm{Ca}^{2+}$ binding by circular dichroism (data not shown). Collectively, these findings, taken together with the rescue experiments, suggest that $\mathrm{C} 2$ domain of $\mathrm{Cc} 2 \mathrm{~d} 1 \mathrm{a}$ is essential for its impact on synaptic transmission although it operates in a $\mathrm{Ca}^{2+}$-independent manner.

\section{DISCUSSION}

In this study, we developed a $\mathrm{KO}$ mouse model to investigate the function of $\mathrm{Cc} 2 \mathrm{~d} 1 \mathrm{a}$, a $\mathrm{C} 2$ domain protein with unique $\mathrm{NH}_{2}$-terminal DM14 domains. Cc2d1a belongs to a new protein family, and it has another homolog Cc2d1b in mammals. Instead of four DM14 domains at $\mathrm{NH}_{2}$ terminus, $\mathrm{Cc} 2 \mathrm{~d} 1 \mathrm{~b}$ (mouse) has three DM14 and shares $43 \%$ identity with Cc2d1a (mouse) on the basis of amino acid sequences. Our experiments demonstrated that Cc2d1a is essential for survival, 


\section{A 7 days in vitro}
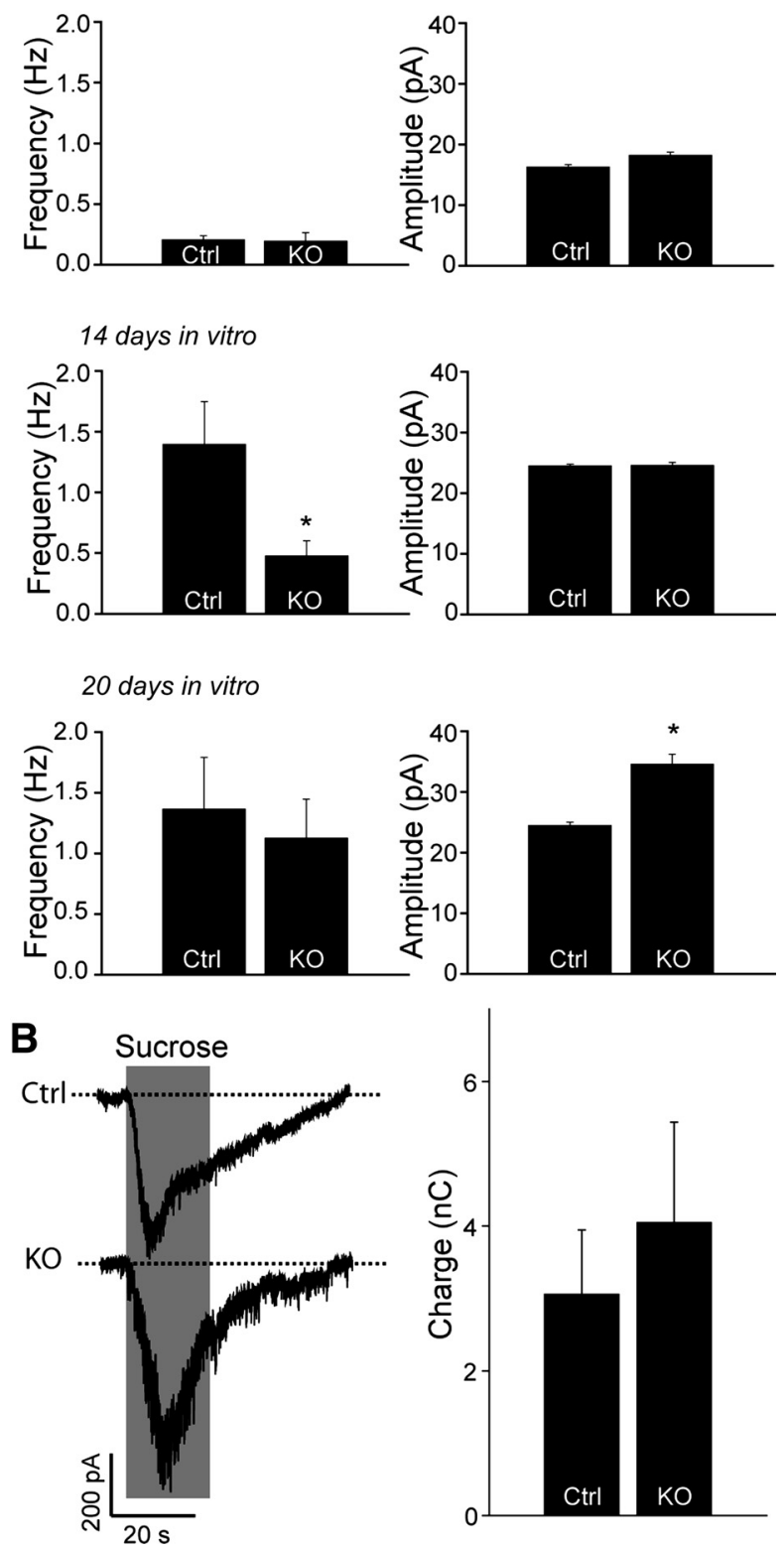

Fig. 6. Analysis of spontaneous release events and hypertonic sucrose-triggered responses of GABAergic synapses in Cc2d1a-deficient neurons. A: cultured cortical neurons at 7 (top), 14 (middle), and 21 (bottom) DIV were analyzed in voltage-clamp mode in the presence of $1 \mu \mathrm{M}$ tetrodotoxin. Comparison of miniature IPSC (mIPSC) frequencies (left) and amplitudes (right) for GABAergic events in control and Cc2d1a-deficient neurons (7 DIV: Control, $n=8$ neurons, KO, $n=10$; 14 DIV: Control, $n=16$ KO, $n=11 ; 21$ DIV: Control, $n=7, \mathrm{KO}, n=5) *$ Significant differences between control and $\mathrm{KO}$ cells. $B$ : inhibitory responses evoked by hypertonic sucrose $(+500 \mathrm{mOsm})$ application in control and Cc2d1a-deficient synapses (left). Sample recordings of inhibitory synaptic responses to a local application of $+500 \mathrm{mM}$ sucrose in Tyrode solution in the presence of $1 \mathrm{mM}$ tetrodotoxin (right). The average values of total release induced by hypertonic sucrose application did not reveal a difference between control and $\mathrm{KO}$ conditions (Control, $n=3$ neurons; $\mathrm{KO}, n=4$ ). The total charge transfer triggered by hypertonic sucrose application was determined by integrating the area over the first $10 \mathrm{~s}$ of 20 -s stimulation.

indicating that $\mathrm{Cc} 2 \mathrm{~d} 1 \mathrm{a}$ and $\mathrm{Cc} 2 \mathrm{~d} 1 \mathrm{~b}$ serve critical and nonredundant functions.

Cc2d1a-deficient animals have beating hearts at birth, but they do not breathe on their own and soon die. As mentioned in the introduction, the Drosophila orthologs of Cc2d1a regu- lates Notch activation. Notch is expressed at a particular high level in the thymus and regulates T-cell development (Rothenberg and Taghon 2005). We analyzed the expression of CD4 and CD8 on thymocytes by fluorescence-activatedcell sorting and found no difference between control and KO mice at E18.5 (data not shown). We also observed similar responses to stimuli from control and KO thymocytes of E18.5 littermate embryos. Thus we were unable to detect any impairment in the development and function of thymocytes, suggesting that Notch pathway is intact in the absence of Cc2dla.

In the analysis of $\mathrm{Cc} 2 \mathrm{~d} 1 \mathrm{a}$-deficient mice, we focused on the brain for several reasons. First, the brain expresses the highest level of Cc2d1a among all the tissues tested (thymus, lung, heart, spleen, liver, and kidney). In situ hybridization shows that $\mathrm{Cc} 2 \mathrm{~d} 1 \mathrm{a}$ mRNA is ubiquitous in the brain and enriched in the gray matter, including cortical plate. Second, loss-offunction mutations in CC2D1A gene in humans have been associated with mental retardation, suggesting that CC2D1A may impact neuronal functions. Third, perinatal lethality has been observed upon deleting some genes that regulate neurotransmitter release and synaptic functions. These include
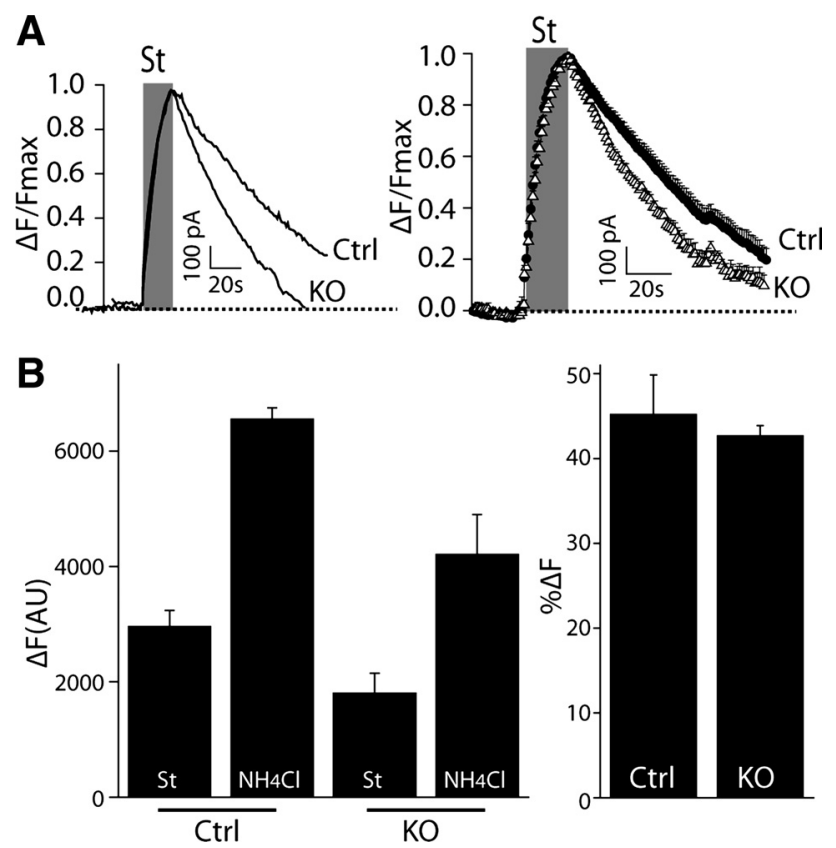

Fig. 7. Analysis of synaptic vesicle recycling using Synaptophysin-pHluorin. Cultured cortical neurons were infected with lentiviruses expressing synaptophysin-pHluorin at 4 DIV and analyzed at 14 DIV. A: left: representative experiment. Right: averaged traces (Control, $n=12$ experiments; KO, $n=8$ ). Electrical stimulation was applied at $20 \mathrm{~Hz}$ during $20 \mathrm{~s}$. St, stimulation. Fluorescence changes were normalized to the peak value. The magnitude of the fluorescence change during stimulation represents the amount of synaptophysin-pHluorin accumulated on the synaptic plasma membrane. Fluorescence decay after stimulation represents endocytic retrieval of synaptophysin-pHluorin and reacidification of vesicles. $\Delta \mathrm{F}=\mathrm{F}_{0}$ (resting fluorescence)- Fmax (peak fluorescence). The average values are statistically different $(P<0.05)$ after the peak is reached, denoting a faster endocytosis in the KO neurons. $B$ : left: fluorescence changes over baseline $\left(\mathrm{F}_{0}\right)$ during stimulation or $\mathrm{NH}_{4} \mathrm{Cl}(50$ $\mathrm{mM}$ ) treatment do not show a statistically significant difference between control and KO synapses. AU, arbitrary units; Control, $n=5$ experiments; $\mathrm{KO}, n=4$. Right: relative changes in fluorescence during stimulation with respect to the change after $\mathrm{NH}_{4} \mathrm{Cl}$ treatment is not different between the 2 groups. These data suggest that the number of vesicles that contain synaptophysin-pHluorin as well as the recycling fraction are not different between control and $\mathrm{KO}$ synapses despite a clear difference in the rate of endocytosis. 
A

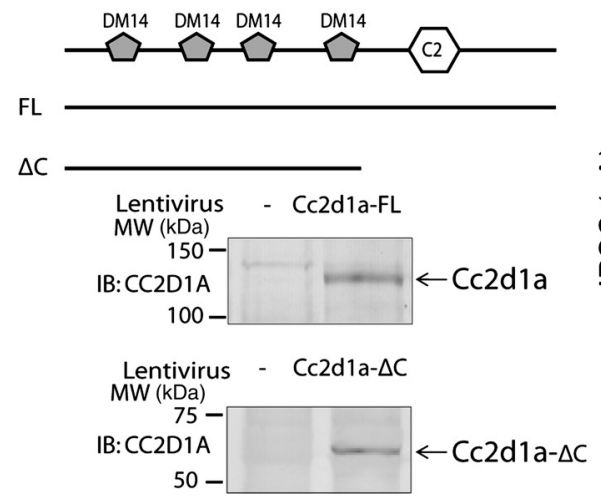

B

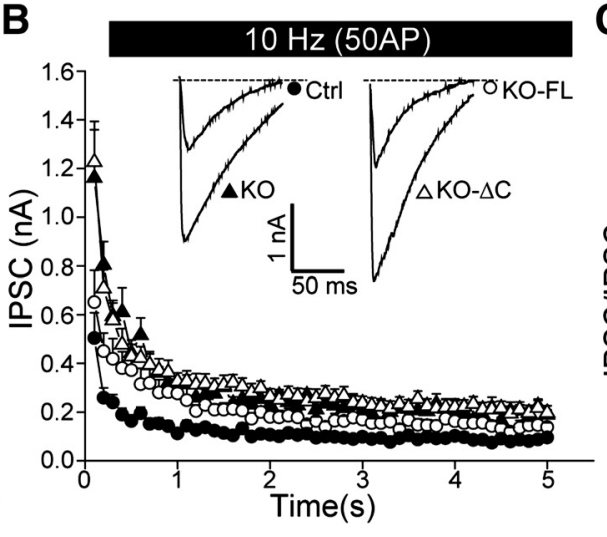

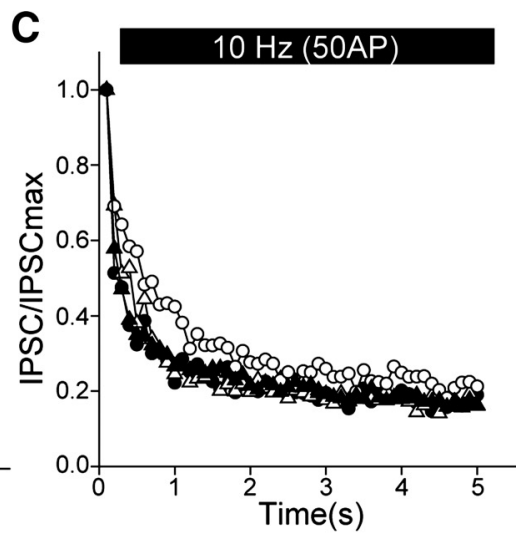

Fig. 8. Cc2d1a partially rescues IPSCs in Cc2d1a-deficient neurons. A: cortical cultures were infected at 4 DIV with lentiviruses expressing either full-length (FL) or $\mathrm{COOH}$-terminal-deletion mutant of Cc2d1a. The expression of exogenous proteins was examined by immunoblotting using CC2D1A-specific antibody. $B$ : IPSC was examined at 14 DIV as described in Fig. 4 (Control, $n=17$ neurons; KO, $n=15$; KO-FL, $n=14$; KO- $\Delta$ C, $n=8$ ). We used ANOVA 1-way and Bonferroni or Fisher test to compare 4 groups of neurons. Expression of FL Cc2d1a in KO neurons significantly rescued IPSCs in response to the first and second action potential stimulation in the train compared with control neurons. The difference in evoked responses was not significant after the third action potential and beyond indicating partial rescue. However, $\Delta \mathrm{C}-\mathrm{Cc} 2 \mathrm{~d} 1 \mathrm{a}$ construct did not rescue IPSCs throughout the stimulation train under all experimental conditions. $C$ : graph depicts normalized IPSC amplitudes under all conditions. Depression kinetics did not reveal a significant difference.

components of the core release machinery such as synaptobrevin-2, $S$-nitroso- $N$-acetyl penicillamine-25, Munc-18, and synaptotagmin-1 (Geppert et al. 1994; Verhage et al. 2000; Schoch et al. 2001; Washbourne et al. 2002). In many cases, although the targeted gene is essential for proper brain function, knocking out the gene does not impair normal assembly of brain structures (Verhage et al. 2000). In the case of Cc2d1a $\mathrm{KO}$, we did not detect any anatomical defects in the heart, lung, muscle, or brain or the innervation of diaphragm muscles. However, we cannot exclude subtle alterations in the development of these organs in the absence of $\mathrm{Cc} 2 \mathrm{~d} 1 \mathrm{a}$, which could contribute to the lethal phenotype.

To circumvent early lethality in $\mathrm{KO}$ animals, we took advantage of an in vitro culture system in which embryonic neurons mature and develop extensive synaptic connections and the basal properties of $\mathrm{Cc} 2 \mathrm{~d} 1 \mathrm{a}$-deficient neurons can be studied electrophysiologically (Schoch et al. 2001). We chose cortical neurons because the expression of $\mathrm{Cc} 2 \mathrm{~d} 1 \mathrm{a}$ is enriched in the cortex on the basis of mRNA expression pattern (Fig. 2).

Our analysis of synaptic transmission between cortical neurons revealed several key differences in the properties of synaptic transmission between neurons obtained from agematched litter control and Cc2d1a-deficient embryos. First, loss of $\mathrm{Cc} 2 \mathrm{~d} 1 \mathrm{a}$ causes a robust increase in the size of evoked IPSCs (as well as EPSCs) detected at early phases of synaptic maturation ( 7 to 14 DIV) but not in more mature synapses in 21 DIV. Second, these defects are not matched with parallel deficiencies in the properties of spontaneous miniature release events or release triggered in response to hypertonic sucrose stimulation. Taken together, these observations support the premise that $\mathrm{Cc} 2 \mathrm{~d} 1 \mathrm{a}$ specifically impacts the properties of action potential-evoked $\mathrm{Ca}^{2+}$-dependent neurotransmitter release, presumably by regulating $\mathrm{Ca}^{2+}$-dependent release machinery or possibly by altering coupling to voltage-gated $\mathrm{Ca}^{2+}$ channels. Third, optical imaging of synaptic vesicle exoendocytosis revealed faster compensatory vesicle retrieval, which could augment the potency of evoked neurotransmission during action potential stimulation at steady state (Sara et al. 2002). These optical recordings, however, did not reveal a significant alteration in the ascending phase of the fluorescence signal, which corresponds to surface delivery (balanced with fast retrieval) of the probe during stimulation. This observation indicates that loss of $\mathrm{Cc} 2 \mathrm{~d} 1 \mathrm{a}$ does not impact release dynamics per synapse but augments the number of synapses that respond to stimulation. Indeed, earlier studies have indicated that the ability to respond to fast action potential stimulation is a key step in functional synapse maturation. Before this step, nascent synapses are solely capable of spontaneous release or bulk release in response to massive $\mathrm{Ca}^{2+}$ influx but not rapid action potential-evoked release (Mozhayeva et al. 2002; Shen et al. 2006; Akhtar et al. 2009). All these findings point to a critical role of $\mathrm{Cc} 2 \mathrm{~d} 1 \mathrm{a}$ in developmental regulation of presynaptic function. Nevertheless, our findings do not allow unequivocal distinctions between the two potential functions of Cc2d1a as a transcriptional regulator in the $\mathrm{NF}-\kappa \mathrm{B}$ pathway and a controller of endosomal trafficking (especially as seen in Drosophila). The developmental specificity of our findings to early synapse maturation may point to the $\mathrm{Cc} 2 \mathrm{~d} 1 \mathrm{a}$-dependent transcriptional regulatory process impacting synaptic development akin to recent observations on the negative role of the myocyte enhancer factor-2 family of transcription factors (Flavell et al. 2006; Barbosa et al. 2008) and histone deacetylases 1 and 2 (Akhtar et al. 2009) on synapse maturation. Synaptic maturation is a complex process that is steered by multiple factors, and it is likely that $\mathrm{Cc} 2 \mathrm{~d} 1 \mathrm{a}$ regulates gene transcription of multiple targets through transcription factors such as NF- $\kappa$ B. However, our experiments to date could not identify a significant effect of $\mathrm{Cc} 2 \mathrm{~d} 1 \mathrm{a}$ on $\mathrm{NF}-\kappa \mathrm{B}$-dependent transcriptional regulation in neurons although we cannot exclude more subtle regulatory processes that may have escaped our detection. Our optical imaging experiments, on the other hand, point to a role of $\mathrm{Cc} 2 \mathrm{~d} 1 \mathrm{a}$ in synaptic vesicle endocytosis, which is consistent with its potential role in endosomal and vesicle trafficking processes in Drosophila. At the molecular level our findings indicate that $\mathrm{Cc} 2 \mathrm{~d} 1 \mathrm{a}$ does not bind to $\mathrm{Ca}^{2+}$; thus it cannot act directly as a calcium sensor or a calcium buffer to curtail calcium signaling.

Taken together, our results show that mice deficient in Cc2d1a die at birth, probably because of the impairment of synaptic function in the brain. Our data suggest that endoge- 
nous $\mathrm{Cc} 2 \mathrm{~d} 1 \mathrm{a}$ keeps synaptic maturation in check to make sure that the neuron develops fully functional synapses and responses to stimulations at the right time. Thus, in the absence of $\mathrm{Cc} 2 \mathrm{~d} 1 \mathrm{a}$, the timing of this maturation process is impaired. These results provide some clues to the role of CC2D1A on the nonsyndromic mental retardation phenotype in human patients. Future molecular analysis of Cc2d1a will help us better understand the molecular mechanism underlying the synaptic maturation process and how this cellular defect relates to lethal phenotype in mice and the mental retardation phenotypes in humans.

\section{ACKNOWLEDGMENTS}

We thank the pathology core facility at University of Texas Southwestern for help in histochemical analysis. We thank Drs. Jose Rizo-Rey, Yun Liu, Weichun Lin, Mikhail Khvotchev, Xinran Liu, Thomas C. Südhof, and Nicolai Van Oers for insightful discussions and Ms. Izabela Kornblum for technical support.

\section{GRANTS}

This work was supported by grants from NIH to Z. J. Chen (RO1AI060919) and to E. Kavalali (RO1-MH066198).

\section{DISCLOSURES}

No conflicts of interest, financial or otherwise, are declared by the authors.

\section{REFERENCES}

Agrawal N, Kango M, Mishra A, Sinha P. Neoplastic transformation, and aberrant cell-cell interactions in genetic mosaics of lethal(2)giant larvae (lgl), a tumor suppressor gene of Drosophila. Dev Biol 172: 218-229, 1995.

Akhtar MW, Raingo J, Nelson ED, Montgomery RL, Olson EN, Kavalali ET, Monteggia LM. Histone deacetylases 1, and 2 form a developmental switch that controls excitatory synapse maturation and function. J Neurosci 29: $8288-8297,2009$.

Atasoy D, Schoch S, Ho A, Nadasy KA, Liu X, Zhang W, Mukherjee K, Nosyreva ED, Fernandez-Chacon R, Missler M, Kavalali ET, Sudhof TC. Deletion of CASK in mice is lethal, and impairs synaptic function. Proc Natl Acad Sci USA 104: 2525-2530, 2007.

Barbosa AC, Kim MS, Ertunc M, Adachi M, Nelson ED, McAnally J, Richardson JA, Kavalali ET, Monteggia LM, Bassel-Duby R, Olson EN. $\mathrm{MEF} 2 \mathrm{C}$, a transcription factor that facilitates learning, and memory by negative regulation of synapse numbers and function. Proc Natl Acad Sci USA 105: 9391-9396, 2008.

Basel-Vanagaite L, Attia R, Yahav M, Ferland RJ, Anteki L, Walsh CA, Olender T, Straussberg R, Magal N, Taub E, Drasinover V, Alkelai A, Bercovich D, Rechavi G, Simon AJ, Shohat M. The CC2D1A, a member of a new gene family with $\mathrm{C} 2$ domains, is involved in autosomal recessive non-syndromic mental retardation. J Med Genet 43: 203-210, 2006.

Buratovich MA, Bryant PJ. Duplication of 1(2)gd imaginal discs in Drosophila is mediated by ectopic expression of wg, and dpp. Dev Biol 168: 452-463, 1995.

Buratovich MA, Bryant PJ. Enhancement of overgrowth by gene interactions in lethal(2)giant discs imaginal discs from Drosophila melanogaster. Genetics 147: 657-670, 1997.

Childress JL, Acar M, Tao C, Halder G. Lethal giant discs, a novel $\mathrm{C} 2$-domain protein, restricts notch activation during endocytosis. Curr Biol 16: 2228-2233, 2006.

Flavell SW, Cowan CW, Kim TK, Greer PL, Lin Y, Paradis S, Griffith EC, Hu LS, Chen C, Greenberg ME. Activity-dependent regulation of
MEF2 transcription factors suppresses excitatory synapse number. Science 311: $1008-1012,2006$.

Gallagher CM, Knoblich JA. The conserved c2 domain protein lethal (2) giant discs regulates protein trafficking in Drosophila. Dev Cell 11: 641653, 2006.

Geppert M, Goda Y, Hammer RE, Li C, Rosahl TW, Stevens CF, Sudhof TC. Synaptotagmin I: a major $\mathrm{Ca}^{2+}$ sensor for transmitter release at a central synapse. Cell 79: 717-727, 1994.

Giloh H, Sedat JW. Fluorescence microscopy: reduced photobleaching of rhodamine, and fluorescein protein conjugates by $n$-propyl gallate. Science 217: 1252-1255, 1982.

Harper RM, Gozal D, Bandler R, Spriggs D, Lee J, Alger J. Regional brain activation in humans during respiratory, and blood pressure challenges. Clin Exp Pharmacol Physiol 25: 483-486, 1998.

Jaekel R, Klein T. The Drosophila Notch inhibitor, and tumor suppressor gene lethal (2) giant discs encodes a conserved regulator of endosomal trafficking. Dev Cell 11: 655-669, 2006.

Kavalali ET, Klingauf J, Tsien RW. Activity-dependent regulation of synaptic clustering in a hippocampal culture system. Proc Natl Acad Sci USA 96: 12893-12900, 1999.

Khvotchev MV, Sudhof TC. Stimulus-dependent dynamic homo-, and heteromultimerization of synaptobrevin/VAMP and synaptophysin. Biochemistry 43: 15037-15043, 2004.

Klein T. The tumour suppressor gene 1(2)giant discs is required to restrict the activity of Notch to the dorsoventral boundary during Drosophila wing development. Dev Biol 255: 313-333, 2003.

Mozhayeva MG, Sara Y, Liu X, Kavalali ET. Development of vesicle pools during maturation of hippocampal synapses. J Neurosci 22: 654-665, 2002.

Nakamura A, Naito M, Tsuruo T, Fujita N. Freud-1/Aki1, a novel PDK1interacting protein, functions as a scaffold to activate the PDK1/Akt pathway in epidermal growth factor signaling. Mol Cell Biol 28: 5996-6009, 2008

Ou XM, Lemonde S, Jafar-Nejad H, Bown CD, Goto A, Rogaeva A, Albert PR. Freud-1: A neuronal calcium-regulated repressor of the 5-HT1A receptor gene. J Neurosci 23: 7415-7425, 2003.

Rizo J, Sudhof TC. C2-domains, structure, and function of a universal $\mathrm{Ca}^{2+}$-binding domain. J Biol Chem 273: 15879-15882, 1998.

Rogaeva A, Ou XM, Jafar-Nejad H, Lemonde S, Albert PR. Differential repression by Freud-1/CC2D1A at a polymorphic site in the dopamine-D2 receptor gene. J Biol Chem 282: 20897-20905, 2007.

Rothenberg EV, Taghon T. Molecular genetics of T cell development. Anпи Rev Immunol 23: 601-649, 2005.

Sara Y, Mozhayeva MG, Liu X, Kavalali ET. Fast vesicle recycling supports neurotransmission during sustained stimulation at hippocampal synapses. $J$ Neurosci 22: 1608-1617, 2002.

Shen W, Wu B, Zhang Z, Dou Y, Rao ZR, Chen YR, Duan S. Activityinduced rapid synaptic maturation mediated by presynaptic cdc42 signaling. Neuron 50: 401-414, 2006.

Schoch S, Deak F, Konigstorfer A, Mozhayeva M, Sara Y, Sudhof TC, Kavalali ET. SNARE function analyzed in synaptobrevin/VAMP knockout mice. Science 294: 1117-1122, 2001.

Vega RB, Matsuda K, Oh J, Barbosa AC, Yang X, Meadows E, McAnally J, Pomajzl C, Shelton JM, Richardson JA, Karsenty G, Olson EN. Histone deacetylase 4 controls chondrocyte hypertrophy during skeletogenesis. Cell 119: 555-566, 2004.

Verhage M, Maia AS, Plomp JJ, Brussaard AB, Heeroma JH, Vermeer H, Toonen RF, Hammer RE, van den Berg TK, Missler M, Geuze HJ, Sudhof TC. Synaptic assembly of the brain in the absence of neurotransmitter secretion. Science 287: 864-869, 2000.

Washbourne P, Thompson PM, Carta M, Costa ET, Mathews JR, LopezBendito G, Molnar Z, Becher MW, Valenzuela CF, Partridge LD, Wilson MC. Genetic ablation of the t-SNARE SNAP-25 distinguishes mechanisms of neuroexocytosis. Nat Neurosci 5: 19-26, 2002.

Zhao M, Li X, Chen Z. CC2D1A, a DM14, C2 domain protein, activates NF[kappa]B through the canonical pathway. J Biol Chem 285: $24372-$ 24380, 2010

Zhu Y, Xu J, Heinemann SF. Two pathways of synaptic vesicle retrieval revealed by single-vesicle imaging. Neuron 61: 397-411, 2009. 